\title{
Evaluation of Type of Nephropathy in Patients of Type-2 Diabetes Mellitus
}

\author{
S. Nayak1, S. K. Tripathy¹, S. Das', B. P. Das², C. R. Kar³ \\ ${ }^{1}$ Department of Medicine, SCB Medical College, Cuttack, India \\ ${ }^{2}$ Department of Pathology, SCB Medical College, Cuttack, India \\ ${ }^{3}$ Department of Nephrology, SCB Medical College, Cuttack, India \\ Email: sarojtripathy1@hotmail.com
}

How to cite this paper: Nayak, S., Tripathy, S.K., Das, S., Das, B.P. and Kar, C.R. (2017) Evaluation of Type of Nephropathy in Patients of Type-2 Diabetes Mellitus. Journal of Diabetes Mellitus, 7, 281-293. https://doi.org/10.4236/jdm.2017.74023

Received: September 16, 2017

Accepted: October 23, 2017

Published: October 26, 2017

Copyright (๑) 2017 by authors and Scientific Research Publishing Inc. This work is licensed under the Creative Commons Attribution International License (CC BY 4.0).

http://creativecommons.org/licenses/by/4.0/

(c) (i) Open Access

\begin{abstract}
Background of the Study, Aims and Objectives: There are very few studies on histological patterns of diabetic nephropathy in our part of country. The aim of this study was to evaluate the renal involvement in patients with Type-2 diabetes mellitus (T2DM), assess the histopathological changes and establish a clinico-pathological correlation. Subjects, Method and Materials: Thirty two Type $2 \mathrm{DM}$ patients with nephropathy, after screening consecutive hundred(100) Type 2 Diabetics admitted to the Medicine Department were evaluated for renal involvement by kidney biopsy and histopathological study. Statistical analysis was done by student's t-test, chi-square and linear regression analysis. Results: Thirty two patients (32) with diabetic nephropathy (20 males and 12 females) formed the study group out of hundred (100) consecutive Type- 2 diabetes mellitus patients (58 males and 42 females) admitted to Medicine Department of SCB Medical College Hospital, Cuttack. The frequency of occurrence of clinical diabetic nephropathy was $32 \%$. Most of the patients were having duration of DM of 6-10 years (87.5\%). Pedal edema was found in $96.87 \%$, hypertension in $87.5 \%$ patients respectively. Regression analysis showed that durations of DM and $\mathrm{HbAlc}$ were the two significant risk factors $(\mathrm{P}<0.05)$ for development of nephropathy. Histopathologically, diffuse glomerulosclerosis was the most common form of renal abnormality found in $93.75 \%$ followed by nodular glomerulosclerosis in $62.50 \%$ with overlap in many patients, membranous nephropathy in $12.5 \%$ and focal necrotising glomerulonephritis in $6.25 \%$ respectively. There was no statistically significant clinicopathological correlation observed between clinical and biochemical parameters in patients harbouring the two predominant histological types of nephropathy i.e. diffuse and nodular glomerulosclerosis with respect to age, sex, duration of diabetes, body mass index, systolic blood pressure, HbA1c, 24 hour urinary protein, creatinine clearance, serum urea, serum
\end{abstract}


creatinine or lipid profile. Conclusion: Durations of diabetes and $\mathrm{HbAlc}$ were found to be strongly associated with development of diabetic nephropathy. Diffuse glomerulosclerosis was the most common form of renal abnormality found in $93.75 \%$ followed by nodular glomerulosclerosis in $62.50 \%$ of patients.

\section{Keywords}

Type 2 DM, Diabetic Nephropathy, Renal Biopsy, Diffuse Glomerulosclerosis, Nodular Glomerulosclerosis

\section{Introduction}

The global burden of DM is enormous with an estimated 366 million people living with DM worldwide (2011) [1]. India accounted for nearly one sixth of global diabetes burden in 2011 with about 62 million of people affected by diabetes which is projected to rise to 101 million by 2030 [1] [2]. Type 2 diabetes mellitus (T2DM) is most prevalent form of DM seen in India and constitute more than 95\% of the diabetes population [2] [3] [4]. The chronic hyperglycemia associated with disturbance in fat and protein metabolism, if not treated adequately can lead to long term vascular complications as well as acute metabolic complications, susceptibility to infection and non-alcoholic fatty liver disease [5] [6] [7] [8].

Diabetes related vascular complications can be broadly classified as Microvascular complications which affect retina (Diabetic retinopathy), kidney (Diabetic nephropathy) and the peripheral nerves (Diabetic neuropathy) or macrovascular complications which includes coronary artery disease, cerebrovascular disease and peripheral vascular disease. Premature morbidity and mortality in diabetes occurs due to these complications. Diabetic nephropathy is the leading cause of End Stage Renal Disease (ESRD) worldwide [5].

Early work for diabetic renal disease in India was done under the aegis of World Health Organisation (WHO) in 1975 to 1978. In this Multi National Study of Diabetic Vascular Disease (M.N.S.D.V.D) spanning fourteen countries with India as one of the centres (Delhi) observed DN in 9.3\% males and $4.2 \%$ females with DM duration of 0 - 6 years, in $10.7 \%$ males and $5.7 \%$ females with DM duration of 6 to 13years and in 23\% males and 13.6\% females with DM duration of $\geq 14$ years [9]. Data presented in Table 1 .

Significant albuminuria or serum creatinine $>1.5 \mathrm{mg} / \mathrm{dl}$.

Following the same method Indian Council of Medical Research (ICMR) conducted a study in nine centres in India in which SCB Medical College \& Hospital, Cuttack was one of the centre. DN in this study was observed in $12.2 \%$ of males and $5.3 \%$ of females in the first group (DM duration of $0-6$ years), in $18 \%$ males and $8.7 \%$ females in the second group (DM duration of $6-13$ years) and in $22.5 \%$ males and $7.5 \%$ of females in the third group (DM duration $\geq 14$ 
Table 1. Diabetes renal disease [9].

\begin{tabular}{|c|c|c|c|c|c|c|c|}
\hline \multicolumn{4}{|c|}{ W.H.O.M.N.S.V.D. (1975-78) } & \multicolumn{4}{|c|}{ ICMR (1986-89) } \\
\hline \multicolumn{4}{|c|}{14 Countries } & \multicolumn{4}{|c|}{9 Centres } \\
\hline \multicolumn{4}{|c|}{ (Delhi Centre) } & & & & \\
\hline Duration (Yrs) & $0-6$ & $7-13$ & $\geq 4$ & Duration (yrs) & $0-6$ & $7-13$ & $\geq 14$ \\
\hline$M(n-289)$ & 9.3 & 10.7 & 23.0 & $M(n=262)$ & 12.2 & 18.0 & 22.5 \\
\hline $\mathrm{F}(\mathrm{n}-266)$ & 4.2 & 5.7 & 13.6 & $\mathrm{~F}(\mathrm{n}=245)$ & 5.3 & 8.7 & 7.5 \\
\hline
\end{tabular}

Table 2. Mortality data. AIIMS—causes of death (percent) amongst diabetics in the last 3 decades [9].

\begin{tabular}{cccc}
\hline \multirow{2}{*}{ Cause of Death } & 1966 & 1976 & 1986 \\
\cline { 2 - 4 } & $\mathrm{n}=75$ & $\mathrm{~N}=560$ & $\mathrm{n}=580$ \\
\hline DKA & 9.3 & 12.4 & 6.5 \\
Total Vascular Disease & 76.0 & 69.0 & 74.5 \\
Cardiac & 42.3 & 29.1 & 23.8 \\
Cereberal & 11.0 & 11.7 & 10.5 \\
Renal & 22.7 & 28.2 & 40.2 \\
Cirrhosis & 6.7 & 3.2 & 3.3 \\
Infections & 2.7 & 5.5 & 9.2 \\
Others & 4.0 & 9.9 & 6.5 \\
\hline
\end{tabular}

years) [9]. Another study in All India Institute of Medical Sciences (AIIMS), Delhi analysed the mortality amongst diabetics from 1966-86 (three decades) and observed that death due to renal cause was $22.7 \%$ in $1966,28.2 \%$ in 1976 and $40.2 \%$ in 1986 respectively [9]. Data presented in Table 2. A study from Cuttack has revealed DN as the cause of death in $17 \%$ and $35 \%$ of cases in T2DM patients in 1977 and 1988 respectively [6]. Nephropathy affects 20-30\% of T2DM patients according to western studies [10].

Pioneering epidemiological work done by Indian CKD Registry established under the aegis of Indian Society of Nephrology(ISN) had made pertinent observations that diabetes mellitus is the cause of CKD in 31.2\% of patients [11]. Screening and Early Evaluation of Kidney Disease (SEEK) study reported prevalence of CKD to be $17.4 \%$ (urban $25.5 \%$ vs rural 9.4\%).

Study in Cuttack showed that there was greater degree of insulin resistance and $\beta$ cell dysfunction and atherosclerosis in diabetics than non-diabetics with chronic kidney disease [12]. Another study from the same institute has revealed that proteinuria is common and more related to glycemic status. Microalbuminuria in Type-2DM patients found to be a marker of generalised vascular endothelial dysfunction in the same study [12]. Indian Council of Medical Research (ICMR) study had revealed nephropathy in $15.4 \%$ males and $13.9 \%$ of females [13]. 
The Chennai Urban Rural Epidemiological Study (CURE) revealed overt proteinuria in $2.2 \%$ and microalbuminuria in $26.9 \%$ of population [14]. The Chunampet Rural Diabetes Prevention Project (CRDPP) study revealed the prevalence of diabetic nephropathy to be $24.3 \%$ [3]. Study from Bikaner and North Delhi (Clinic Based) revealed the prevalence of diabetic nephropathy to be $26.8 \%$ and $15.3 \%$ respectively [7] [8]. According to CINDI study (Multicentric Clinic Based), the prevalence of diabetic nephropathy in newly detected diabetes mellitus is $13.15 \%$ [15].

Till date there are few studies regarding different types of nephropathy in patients of Type-2 Diabetes Mellitus. Hence the present study was conducted to evaluate renal involvement in patients of Type-2 diabetes mellitus.

\section{Aim of the Study}

This study was carried out to determine various renal histopathological lesions in Type-2 diabetis mellitus patients with renal dysfunction and to establish clinicpathological correlation.

\section{Materials and Methods}

Hundred (100) Type-2 DM patients consecutively admitted to PG Department of Medicine of S.C.B. Medical College and Hospital, Cuttack were evaluated for presence of nephropathy. Out of hundred (100) consecutive Type-2 DM patients 32 cases were found to have nephropathy as per the inclusive criteria of macrolabuminuria $(>300 \mathrm{mg} / \mathrm{dl})$ and/or serum creatinine $>1.2 \mathrm{mg} / \mathrm{dl}$ [5]. They were enrolled for the study, out of which 20 were males and 12 were females. The study had Institute Ethical Committee (IEC) approval.

All T2DM patients with nephropathy of both genders having age more than 30 year were included in the study while patients with Type 1 Diabetes, Gestational Diabetes, Secondary Diabetes, patients with HIV infection, patients on steroid therapy, patients on chronic use of nephrotoxic drugs, solitary kidney on ultrasound of abdomen and pelvis, deranged coagulation profile, thrombocytopenia, uncontrolled hypertension, urinary tract infection and obstructive uropathy were excluded from the study.

Detail clinical evaluation was done in all cases. Laboratory investigation like complete blood count $(\mathrm{CBC})$, urine routine and microscopic, urine culture, fasting blood glucose, $2 \mathrm{hr}$ postprandial blood glucose, $\mathrm{HbAlc}$, serum urea and creatinine, 24 hour urinary protein, serum sodium and potassium, liver function test, lipid profile, ECG and ultrasound of abdomen and pelvis were done after obtaining fully informed written consent.

Renal biopsy was done by nephrologist in the department of nephrology after recieving proper consent from the patients in S.C.B. Medical College Hospital, Cuttack. Kidney biopsy was performed with real-time ultrasound guidance and disposable automated biopsy needles. We used 18-gauge needles for greater tissue yield and fewer bleeding complications. 
The tissue core was examined under light microscope to ensure that renal cortex had been obtained. A second pass of the needle was usually performed to obtain additional tissue where ever needed.

Histopathological study was done in Department of Pathology, S.C.B. Medical College, Cuttack. The renal tissue was placed in formalin and $3 \mu \mathrm{m}$ thin, uniform cut sections were used for light microscopy. 10\% aqueous formaldehyde buffer solution was used for fixation for light microscopy. The specimen was stained with hematoxylin and eosin (H\&E), periodic acid-Schiff (PAS) and silver methenamine.

Diabetic nephropathy has been classified into 4 stages based on biopsy studies which are:

\begin{tabular}{ll}
\hline Class I & $\begin{array}{l}\text { Glomerular basement membrane thickening, mild nonspecific changes by } \\
\text { light microscopy }\end{array}$ \\
Class IIa & Mild mesangial expansion \\
Class IIb & Severe mesangial expansion but without nodular sclerosis \\
Class III & Nodular sclerosis \\
Class IV & Advanced diabetic glomerulosclerosis, more than $50 \%$ of glomerulosclerosis. \\
\hline
\end{tabular}

The data were analyzed using SPSS version 18 . For continuous variables, data were presented as the mean \pm standard deviation or the median with range, and the means were compared using one-way analysis of variance. For categorical variables, the data were presented as counts and percentages, and the differences were analyzed using the Chi-square test.

\section{Results}

Thirty two patients (32) with diabetic nephropathy (20 males and 12 females) formed the study group out of hundred (100) Type-2 diabetes mellitus patients (58 males and 42 females) screened. Thus, the frequency of occurrence of clinical diabetic nephropathy was $32 \%$. Majority of patients were from the age group of 50 - 60 years (56.25\%).Most of the patients were having duration of diabetes of 6 - 10 years (87.5\%). Positive family history of diabetes was present in $37.5 \%$ of patients (35\% among males and $41.66 \%$ among females).Most cases of DN (Diabetic Nephropathy) presented with pedal edema as evidenced by 31 (96.87\%) of patients including 19 (59.37\%) males and $12(37.50 \%)$ females had this finding. The mean BMI in the study group was $23.96 \pm 1.93$. Hypertension was present among 28 (87.50\%) of patients including 19 (59.37\%) males and 9 (28.12\%) females. The mean SBP in our study group was $155 \pm 10.31 \mathrm{mmHg}$ and the mean diastolic blood pressure (DBP) was $88.25 \pm 6.88 \mathrm{mmHg}$. The mean GFR and $\mathrm{HbAlc}$ in our study population were $41.64 \pm 19.78 \mathrm{~mL} / \mathrm{min}$ and $8.53 \pm 1.18 \mathrm{re}-$ spectively.

Among risk factors hypertension was present in $87.5 \%$, dyslipidemia in $78.125 \%$ and smoking history in $43.75 \%$ of cases respectively. 
Results of multiple linear regression analysis showed that duration of diabetes and $\mathrm{HbA1C}$ levels are strongly associated with development of nephropathy ( $\mathrm{p}$ value $<0.05$ for each) in diabetes.

Histopathological findings: Out of thirty two diabetic nephropathy patients, sixteen cases gave consent for renal biopsy. The histopathological findings were summarised in Table 3 and presented in Figures 1-3.

\subsection{Glomerular Lesions}

a) Glomerular Obsolescence (global sclerosis): 12(75\%) of the patients had biopsy specimen with varying degrees of glomerular obsolescence (global sclerosis).

Table 3. ABLE.1 Histopathology of renal biopsy.

\begin{tabular}{ccc}
\hline Histopathological lesion & No of cases (16) & Total (100\%) \\
\hline Global sclerosis & 12 & $75 \%$ \\
Diffuse glomerulosclerosis & 15 & $93.75 \%$ \\
Nodular glomerulosclerosis & 10 & $62.5 \%$ \\
Membranous glomerulopathy & 3 & $18.75 \%$ \\
Focal necrotising glomerulonephritis & 1 & $6.25 \%$ \\
Tubular atrophy & 14 & $87.5 \%$ \\
Vacuolation & 10 & $62.5 \%$ \\
Casts & 15 & $93.75 \%$ \\
Interstitial fibrosis & 14 & $87.5 \%$ \\
Hyaline change & 14 & $87.5 \%$ \\
Intimal thickening & 12 & $75.00 \%$ \\
\hline
\end{tabular}

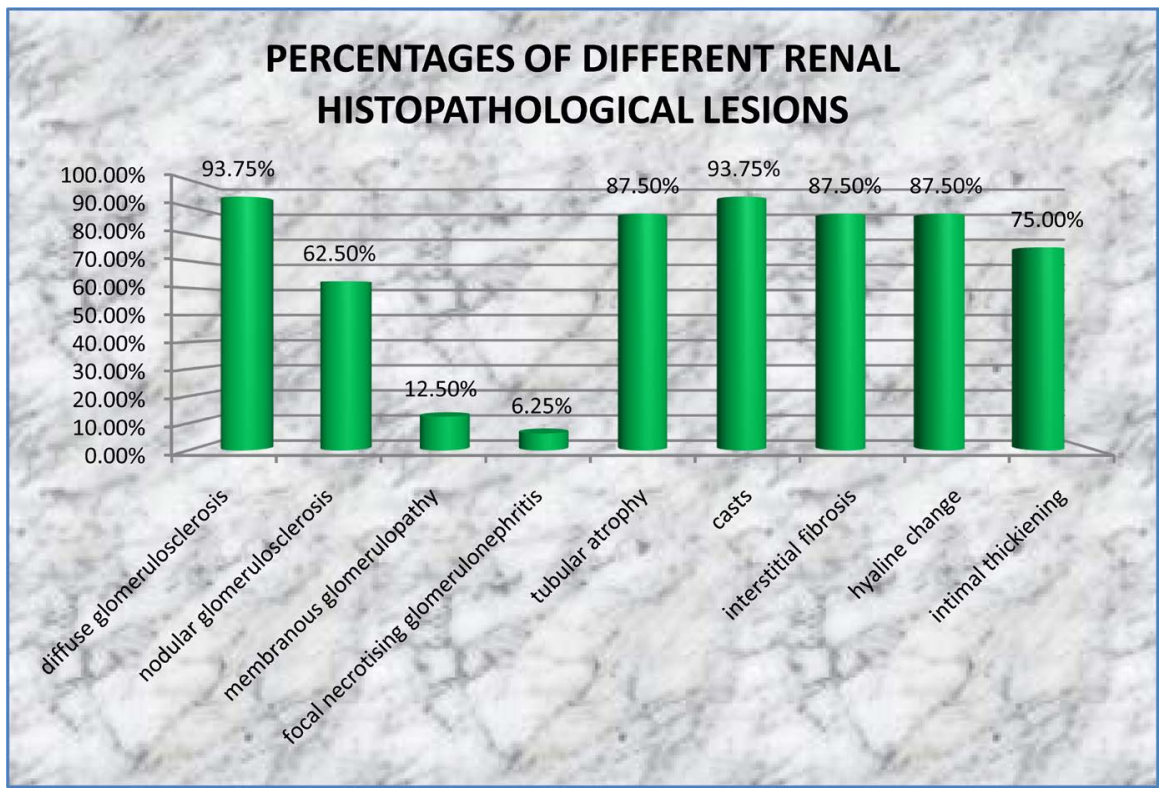

Figure 1. Different histopathological lesions in Kidney biopsy of Type-2DM patients with neprhopathy. 


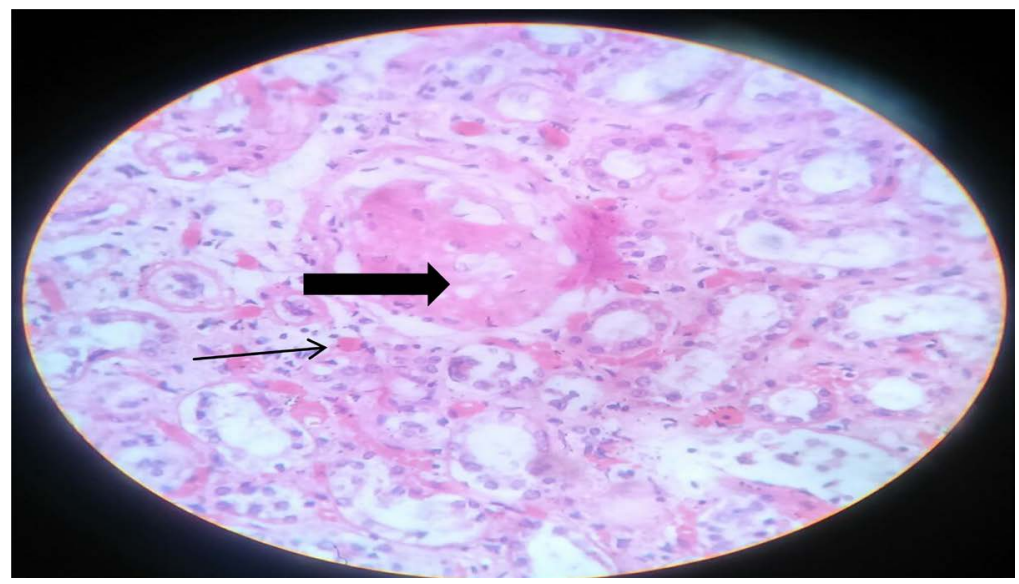

Figure 2. Renal biopsy from a 63 year old Type-2DM patient showing globally sclerosed glomeruli (thick black arrow) and arteriolar hyalinosis (thin black arrow).

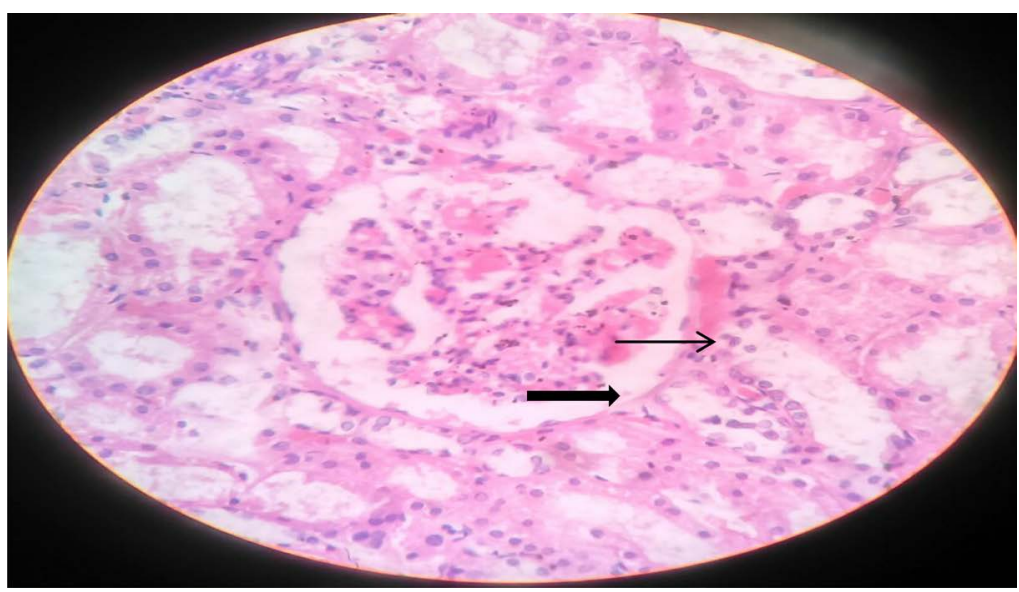

Figure 3. Renal biopsy from a 68 year old male Type-2 DM patient showing nodular glomerulosclerosis (thick black arrow) and basement membrane thickening (thin black arrow).

b) Diffuse glomerulosclerosis (DGS): Diffuse glomerulosclerosis was the most common form of glomerular lesion seen. It was present in $15(93.75 \%)$ patients (13 males and 2 females).

c) Nodular glomerulosclerosis (NGS): Nodular glomerulosclerosis was seen in $10(62.50 \%)$ patients (8 males and 2 females).

d) Non diabetic nephropathy was found in 3 biopsy (18.75\%) of which 2 (12.5\%) were membranous glomerulopathy (MGN) with evidence of segmental tuft sclerosis in 9/35 (25.70\%) of the sampled glomeruli in one patient and 1/9 (11.11\%) in another patient; and 1 (6.25\%) was focal necrotising glomerulonephritis, with partial fibrocellular crescents over 4/26 (15.30\%) glomeruli.

\subsection{Tubular Lesion}

Tubules showed focally prominent cytoplasmic vacuolar change. 10 biopsies (62.50\%) showed tubular vacuolaton. 14 (87.50\%) cases showed tubular atrophy with varying severity. Varying degrees of hyaline and granular casts were ob- 
served in tubules. Casts were present in 15 (93.75\%) of cases.

\subsection{Interstitial Fibrosis}

$14(87.50 \%)$ patients had interstitial fibrosis of varying severity with interstitial inflammation.

\subsection{Vascular Lesion}

Arterioles showed intimal thickening in 12 (75\%) cases and hyalinosis in 14 $(87.50 \%)$ of patients.

The difference in the clinical manifestations between between Diffuse Glomerulosclerosis and Nodular Glomerulosclerosis is presented in Table 4 and the biochemical parameters are given in Table 5 . There was no statistical difference in age, duration of diabetes, BMI as well as syostolic and diastolic blood pressures in both the groups. The gender distribution was also comparable.

As presented in Table 5, the mean HbA1C was more than $9 \%$ in both the groups. The creatine clearence was around $30 \mathrm{ml} / \mathrm{min}$ and was similar in both the groups. Proteinuria of more than $1500 \mathrm{mg} /$ day was observed in both the

Table 4. Clinical data of patients undergone kidney biopsy and diagnosed to be cases DGS and NGS.

\begin{tabular}{cccc}
\hline & Diffuse glomerulosclerosis & Nodular glomerulosclerosis & p-value \\
\hline No of cases & 15 & 10 & 0.9567 \\
Age(years) & $59.4 \pm 8.67$ & $59.6 \pm 9.33$ & 1.000 \\
Sex(M/f) & $13 / 2$ & $8 / 2$ & 0.7267 \\
Duration of diabetes (years) & $9.4 \pm 1.95$ & $9.7 \pm 2.26$ & 0.6113 \\
BMI $\left(\mathrm{kg} / \mathrm{m}^{2}\right)$ & $24.26 \pm 2.60$ & $23.73 \pm 2.39$ & 0.6767 \\
SBP(mm/Hg) & $158.4 \pm 7.79$ & $159.80 \pm 8.61$ & 0.8887 \\
DBP $(\mathrm{mmHg})$ & $87.33 \pm 5.27$ & $87.00 \pm 6.34$ &
\end{tabular}

Table 5. Biochemical-pathological correlation.

\begin{tabular}{|c|c|c|c|}
\hline & Diffuse glomerulosclerosis & Nodular glomerulosclerosis & $\mathrm{p}$-value \\
\hline HBA1c & $9.08 \pm 1.18$ & $9.21 \pm 1.19$ & 0.7904 \\
\hline Creatinine clearance $(\mathrm{ml} / \mathrm{min})$ & $34.83 \pm 17.45$ & $35.649 \pm 19.81$ & 0.9142 \\
\hline Serum urea & $81.46 \pm 42.42$ & $81.70 \pm 51.44$ & 0.989 \\
\hline Creatinine $(\mathrm{mg} / \mathrm{dl})$ & $2.56 \pm 1.004$ & $2.64 \pm 1.173$ & 0.8567 \\
\hline $\begin{array}{l}24 \text { hour urinary } \\
\text { protein (mg/day) }\end{array}$ & $1504 \pm 470.81$ & $1586 \pm 545.20$ & 0.692 \\
\hline Serum triglycerides & $145.00 \pm 36.05$ & $143.80 \pm 29.08$ & 0.931 \\
\hline Total cholesterol & $205.53 \pm 68.16$ & $219.70 \pm 60.28$ & 0.599 \\
\hline LDL cholesterol & $93.00 \pm 20.18$ & $95.30 \pm 23.07$ & 0.794 \\
\hline HDL cholesterol & $45.86 \pm 10.23$ & $44.50 \pm 11.28$ & 0.757 \\
\hline
\end{tabular}


groups. Serum cholesterol levels were higher but mean value of LDLc was below $100 \mathrm{mg} / \mathrm{dl}$ where as HDLc levels were above $40 \mathrm{mg} / \mathrm{dl}$ in both the groups.

\section{Disscussion}

Diabetes mellitus is the commonest metabolic disorder and has a high prevalence in India [8]. Diabetic nephropathy is a clinical hall mark of microangiopathy and is the most important single disorder leading to renal failure in adults [4].

Till date, scanty studies have been conducted regarding the type of nephropathy in T2DM in this part of India, hence this study was conducted to evaluate nephropathy with histopathological changes in T2DM patients.

We found the incidence of diabetic nephropathy to be $32 \%$ in hospitalised patients. It has been reported that among 4837 patients with chronic renal failure seen over a period of 10 years, the prevalence of diabetic nephropathy was 30.3\% India [16]. Other studies have shown prevalence of nephropathy to be $24.3 \%$ in Tamilnadu by CRDPP study [3] and $30.2 \%$ in Bikaner respectively and a crosssectional study from Lucknow in 2012 showed prevalence of diabetic nephropathy to be $20 \%$ [17].

Another study from Karnataka by Raja Reddy et al. [18] in 2012 had reported the prevalence of diabetic nephropathy as $37.02 \%$ (Microalbuminuria was $30.79 \%$ in males and $24.46 \%$ in females and overt nephropathy was $9.27 \%$ in males and $6.73 \%$ in females).

The mean duration of diabetes was $8.25 \pm 1.98$ years in our study. Similar study by Rudberg et al., in a study of adolescents with a mean duration of disease of 10.9 years, found that the duration of disease was an important factor in the overall severity of glomerulopathy [19].

The duration of diabetes, HbAlc level, Systolic and diastolic blood pressure and serum creatinine values are higher in NGS compared to the DGS variety. But there was no statistically significant difference between clinical and biochemical parameters in patients with nodular glomerulosclerosis and diffuse glomerulosclerosis.

The mean duration of diabetes was below $10 \mathrm{yr}$ where as the mean age of patients was below 60 yrs suggesting development of nephropathy occurring at much earlier age in the T2DM in India than described in western literature [5].

The cohurt under study and mean BMI below 25 suggested that obesity is not a deciding factor in T2DM suffering from nephropathy in our population.

Even though majority of patients are in chronic kidney disease stage 3 (CKD stage 3) as per Cr. Clearance the biochemical parameters were worse than expected. (mean Creatinine above $2.5 \mathrm{mg} \%$ and serum urea above $80 \mathrm{mg} \%$ ). This is an observation which is different from that observed in standard literature. The dyslipidemia observed neither revealed low HDLc nor high triglyceride which is classically seen in Indian diabetes. The most important risk factor for development of nephropathy was duration of diabetes followed by hypertension and 
smoking. The CURES-45 reported that risk factors for overt diabetic nephropathy include HBA1C, duration of diabetes, and systolic blood pressure, while for microalbuminuria smoking and diastolic blood pressure were also risk factors [14].

Multiple linear regression analysis of various risk factors with diabetic nephropathy is presented in Table 6. In our study the duration of diabetes, HbAlc, serum urea and HDL cholesterol were strongly associated with development of diabetic nephropathy ( $p$ value of $<0.05$ for each). Similar type of study with regression analysis of risk factors by Agrawal et al. in 2012 from Bikaner, Rajasthan reported that duration of diabetes and $\mathrm{HbAlc}$ were strongly associated with diabetic nephropathy [17].

Histopathologically, DGS was the most predominant form of renal abnormality found in $93.75 \%$ followed by NGS in $62.50 \%$, membranous nephropathy in $12.5 \%$ and focal necrotising glomerulonephritis in $6.25 \%$ of cases respectively. Study by Olsen et al. on 33 biopsies showed that 4 (12.12\%) had Non Diabetic Renal Diseases (NDRD) and remaining 29 (87.87\%) patients had DGS (9 = $27.27 \%)$ and NGS $(20=60.60 \%)$ [20]. Another study by Prakash J. et al. showed prevalence of NDRD in diabetes to be $12.3 \%$. at par with our study [21]. A study by Mathur et al. (1964) showed 71.9\% of DN had DGS and 6.2\% had combined DGS and NGS [22].

M Sahay et al. reported 24.56\% NDRD and 75.43\% DN [23]. DGS and NGS are the two predominant histopathological changes in DN according to western data [24]. Another study by Hirose showed diffuse and nodular lesions in 77.5\%

Table 6. Results of multiple linear regression analysis showing association of various risk factors with diabetic nephropathy dependent variable ( 24 hour urinary protein).

\begin{tabular}{cccc}
\hline Variable & Regression coefficient $\beta$ & P value & 95\% Cl Of coefficient of $\beta$ \\
\hline Age & 0.151 & 0.326 & $-12.199-34.625$ \\
Duration of DM & 0.611 & 0.013 & $39.409-288.190$ \\
SBP & -0.232 & 0.306 & $-0,35.879-11.937$ \\
DBP & 0.017 & 0.901 & $-20.760-23.391$ \\
FBS & -0.029 & 0.898 & $-7.052-6.231$ \\
HbAlc & 0.656 & 0.026 & $39.824-548.531$ \\
Serum urea & -0.609 & 0.035 & $-18.045-0.752$ \\
Serum creatinine & -0.270 & 0.926 & $-190.965-174.681$ \\
GFR & 0.008 & 0.977 & $-15.370-15.799$ \\
BMI & -0.281 & 0.070 & $-161.289-6.999$ \\
CHOL & -0.052 & 0.728 & $-3.366-2.400$ \\
TG & -0.183 & 0.353 & $-7.721-2.910$ \\
HDL & 0.327 & 0.039 & $1.127-39.302$ \\
LDL & 0.140 & 0.432 & $-8.077-18.023$ \\
VLDL & -0.293 & 0.088 & $-40.086-3.067$ \\
\hline
\end{tabular}


and $21.7 \%$ respectively [25]. The findings in our study are comparable to those of earlier studies.

There was not much difference between the clinical and biochemical parameters between patient of DGS and NGS. Similar study by Schwartz et al. in 1998 observed little difference between the clinical and biochemical parameters in these two types of DN patients [26].

\section{Conclusions}

Nephopathy occurs at a much earlier age in T2DM patients in India and the duration of diabetes resulting in nephropathy is less as compared to the western population. The typically described high triglyceride and low HDLc in Indian diabetics were not found in our study.

We conclude that nephropathy in patients of T2DM mellitus is of two distinctive pattern of glomerular pathology i.e. DGS and NGS. There was little difference between clinical and biochemical parameters in the DGS and NGS groups with respect to age, hypertension, BMI, duration of diabetes, dyslipidemia and glycemic control as reflected by HbAlc levels. Also noteworthy in the findings of this study was the fact that glomerular lesions other than those associated with diabetes were found in only three (3) patients. Hence, coexisting non diabetic renal disease may be associated with diabetic nephropathy in only a few patients with T2DM. Studies evaluating renal Parenchymal changes in T2DM are limited. Larger studies undertaking Histopathological evaluation of T2DM with Nephropathy will throw more light on NDRD \& DN.

\section{References}

[1] Unwin, N., whiting, D., Guariguata, L., Ghyoot, G. and Gan, D. (2011) Diabetes Atlas. 5th Edition, International Diabetes Federation, Brussels, 11-74.

[2] Anjana, R.M., Pradeepa, R., Deepa, M., Mohanta, J., Narain, K., Das, H.K., Adhikari, P., Rao, P.V., Sahoo, B., Kumar, A., Bhansali, A., John, M., Luaia, R., Reang, T., Ningombam, S., Jampa, L., Budnah, R.O., Elangovan, N., Subashini, R., Venkatesan, U., Unnikrishnan, R., Das, A.K., Madhu, S.V., Ali, M.K., Pandey, A., Shaliwal, R.S., Kaur, T., Swaminathan, S. and Mohan, V., On Behalf of ICMR-INDIAB Collaborative Study Group (2017) Prevalence of Diabetes and Prediabetes (Impaired Fasting Glucose and/or Impaired Glucose Tolerance) in Urban and Rural India: Phase 1 Results of the Indian Council of Medical Research India Diabetes (ICMR-INDIAB) Study. The Lancet Diabetes and Endocrinology, 54, 3022-3027.

[3] Mohan, V., Deepa, M., Pradeepa, R., Pratibha, V., Datta, M., Sethuraman, R., Rakesh, H., Suchitra, Y., Webster, P., Allender, S., Kaour, A. and Anjana, R.M. (2012) Screening for Diabetes and Its Complications and Providing Diabetes Preventive and Treatment to Rural India through a Combination of Telemedicine and Personalized Care-The Chunampet Rural Diabetes Preventive Project (CRDPP). Journal of Diabetes Science and Technology, 6, 1355-1364.

https://doi.org/10.1177/193229681200600614

[4] Ramachandran, A., Snehalatha, C., Latha, E., Vijay, V. and Viswanathan, M. (1997) Rising Prevalence of NIDDM in an Urban Population in India. Diabetologia, 40, 232-237. https://doi.org/10.1007/s001250050668 
[5] Alvin, C. Powers, Diabetes Mellitus Complications; Obesity, Diabetes Mellitus, and Metabolic Syndrome. 19th Edition, Harrison's Principles of Internal Medicine, 2, 2424-2425.

[6] Das, S., Mishra, R.K. and Jena, B.B. (1991) Mortality Events amongst NIDDM Patients in Orissa. Journal of The Association of Physicians of India, 39, 519-520

[7] Agarwal, R.P., Ola, V., Bishnoi, P., Gothwal, S., Sirohi, P. and Agrawal, R. (2014) Prevalence of Micro and Macrovascular Complications and Their Risk Factors in Type 2 Diabetes Mellitus. Journal of The Association of Physicians of India, 62, 504-508

[8] Chawla, R. (2014) Pathophysiology of Diabetes Complications Indian Perspective. In: Joshi, S., Ed., Medicine Update, The Association of Physicians of India, Vol. 242, 1361-1367.

[9] Ahuja, M.M.S. (1996) Renal Diseases in Diabetes. International Journal of Diabetes in Developing Countries, 16, 117-119.

[10] Molitien, M.E., Defronzo, R.A. and Frenz, M.J. (2004) Nephropathy in Diabetes. Diabetes Care, 27, 579-583.

[11] Rajapurkar, M.M., John, G.T., Kirpalini, A.L., Abraham, G., Agarwal, S.K., Almeida, A.F., Gang, S., Gupta, A., Modi, G., Pahari, D., Pisharody, R., Prakash, J., Raman, A., Rana, D.S., Sharma, R.K., Sahoo, R.N., Sakhuja, V., Tatapudi, R.R. and Jha, V. (2012) What Do We Know about Chronic Kidney Disease in India; First Report of the Indian CKD Registry. BMC Nephrology, 13, 10.

https://doi.org/10.1186/1471-2369-13-10

[12] Das, S., Chandrakanth, M.V., Rout, S.B, Mohanty, R., Singh, S.C., Pattnaik, M. and Sethy, B. (2012) Study of Markers of Atherosclerosis Including Insulin Resistance in Patients with Chronic Renal Failure. Journal of Diabetes Mellitus, 2, 208-213. https://doi.org/10.4236/jdm.2012.22033

[13] Rao, P.V. and Ahuja, M.M.S. (1994) Endocrine Metabolism and Diabetes. Macmillan Publications, Vol. 2, 42-53.

[14] Unnikrishnan, R., Rema, M., Pradeepa, R., Deepa, M., Shanthirani, S., Deepa, R. and Mohan, V. (2007) Prevalence and Risk Factors of Diabetic Nephropathy in an Urban South Indian Population, The Chennai Urban Rural Epidemiology Study (CURES 45). Diabetes Care, 30, 2019-2024. https://doi.org/10.2337/dc06-2554

[15] Sosale, A., Prasanna Kumar, K.M., Sadikot, S.M., Nigam, A., Bajaj, S., Zargar, A.H. and Singh, S.K. (2014) Chronic Complication in Newly Diagnosed Patients with T2DM in INDIA (CINDI). Indian Journal of Endocrinology and Metabolism, 18, 355-360. https://doi.org/10.4103/2230-8210.131184

[16] Mani, M.K. (1998) Patterns of Renalp Disease in Indigenous Populations in India. Nephrology, 4, S4-S7. https://doi.org/10.1111/j.1440-1797.1998.tb00461.x

[17] Mohan, V. (2014) Epidemiology of Complications of Diabetes. In: Chandalia, H.B., Ed., RSSDI Text Book of Diabetes Mellitus, Jaypee Brothers Medical Publishers, 169-183. https://doi.org/10.5005/jp/books/12626_13

[18] Reddy, P.R., Jayarama, N. and Lakshmaiah, V. (2012) Study of Prevalence and Stages of Diabetic Nephropathy in a Rural Tertiary Care Centre-Southern India. Global Journal of Medicine and Public Health, 1, 17-22.

[19] Rudberg, S., Qsterby, R., Dahlquist, G., Nyberg, G. and Persson, B. (1997) Predictors of Renal Morphological Changes in the Early Stage of Microalbuminuria in Adolescents with IDDM. Diabetes Care, 20, 265-271. https://doi.org/10.2337/diacare.20.3.265

[20] Olsen, S. and Mogensen, C.E. (1996) How often Is NIDDM Complicated with 
Non-Diabetic Renal Disease, An Analysis of Renal Biopsies and the Literature. Diabetologia, 39, 1638-1645. https://doi.org/10.1007/s001250050628

[21] Prakash, J., Sen, D. and Kumar, N.S. (2001) Non-Diabetic Renal Disease in Patients with Type 2 Diabetes Mellitus. Journal of Association Physicians of India, 49, 415-420.

[22] Mathur, K.S., Wahi, P.N., Gunta, O.P. and Mathur, C.P. (1964) Diabetic Naphropathy-A Clinical and Histological Study by Renal Biopsy. Journal of Associations of Physicians of India, 12, 535-546.

[23] Sahay, M., Mahankali, R.K., Ismal, K., Vali, P.S., Sahay, R.K. and Swarnalata, G. (2014) Renal Histology in Diabetic Nephropathy: A Novel Perspective. Indian Journal of Nephrology, 24, 226-231.

[24] Maitra, A. (2014) Diabetic Nephropathy, Robbin's and Cotran's Pathologic Basis of Diseases. South Asian Edition, Vol. 2, 1118-1119.

[25] Hirose, K. (1970) Clinical and Pathological Features of Diabetic Nephropathy in Japanese Diabetic Patients. In: Diabetes Mellitus in Asia, Excerpta Medica, 177-182.

[26] Schwartz, M.M., Lewis, E.J., Martin, T.L., Lewis, J., Batlle, D. and The Collaborative Study Group (1998) Renal Pathology Patterns in Type 2 Diabetes Mellitus: Relationship with Retinopathy. Nephrolology Dialysis Transplantation, 13, 2547-2552. https://doi.org/10.1093/ndt/13.10.2547 